\title{
Failure to Read Input Signal
}

National Cancer Institute

\section{Source}

National Cancer Institute. Failure to Read Input Signal. NCI Thesaurus. Code C63164.

Problem associated with a failure of the device to read a signal for interpretation or measurement. 